\title{
PERFIL DOS PACIENTES PEDIÁTRICOS AVALIADOS PELA RESIDÊNCIA MULTIPROFISSIONAL EM UM HOSPITAL UNIVERSITÁRIO
}

\section{PROFILE OF PATIENTS PEDIATRICS SAMPLED AT THE MULTIPROFESSIONAL RESIDENCY IN AN UNIVERSITY HOSPITAL}

\section{PERFIL DE PACIENTES PEDIÁTRICOS EVALUADOS POR LA RESIDENCIA MULTIPROFESIONAL EN UN HOSPITAL UNIVERSITARIO}

Recebido: 12/09/2014

Aprovado: 06/06/2015

Danielle Mendonça Araujo ${ }^{1}$
Nayara Bernardes Segava $^{2}$
Fernanda Godoi de Paula $^{3}$
Lorena Candelori Vidal $^{4}$
Juliana Cançado Moraes $^{5}$
ordana Moreira de Almeida
Ana Paula Espíndula

Com o objetivo de analisar o perfil dos pacientes internados na unidade de pediatria de um hospital universitário, realizou-se um estudo descritivo e retrospectivo que incluiu crianças internadas nas Enfermarias de Pediatria e no Pronto Socorro Infantil, de um hospital universitário. Os dados foram obtidos a partir dos registros das avaliações realizadas de maio a outubro de 2012. Foram avaliadas 404 crianças, dessas 35\% são lactentes e $59 \%$ do sexo masculino. Maio foi o mês de maior incidência de internações (21,5\%) e 58\% eram crianças procedentes de Uberaba. Quanto ao número de moradores, teve-se uma frequência de quatro pessoas/casa (31,6\%), sendo a renda de dois salários mínimos $(33,6 \%)$ e, do total de crianças, 38\% recebem algum tipo de benefício. As doenças com maior ocorrência estão relacionadas ao aparelho respiratório (28,2\%). Os dados permitiram caracterizar a amostra estudada, compreender o perfil do adoecimento nessa faixa etária, bem como, elaborar planos de atenção à saúde que previnam o agravamento das doenças a fim de se evitarem hospitalizações futuras, sugerindo o enfoque da prevenção de acometimentos respiratórios na atenção primária.

Descritores: Perfil de Saúde; Unidades de Internação; Criança.

Aiming to analyze the profile of patients admitted to the pediatric unit of a university hospital, it performed a descriptive, retrospective study that included children hospitalized in Pediatric Wards and Infant Emergency Room, of universitario hospital. Data was obtained from the records of evaluations carried out from May to October 2012.404 children were evaluated, among which $35 \%$ were infants and $59 \%$ were male. May was the month of highest incidence of hospitalizations (21.5\%) and 58\% were children from Uberaba, Minas Gerais, Brazil. As for the number of residents, it had a frequency of four people/household (31.6\%), with the prevalence of income of two minimum wages $(33.6 \%)$ and among the total number of children, 38\% receive some form benefit. The most occurring diseases are related to the respiratory tract $(28.2 \%)$. The data allow us to characterize the sample group, understand the illness profile, as well as develop plans of health attention that prevent the aggravation of the diseases, in order to avoid hospitalizations future, suggesting the focus on prevention of breathing issues through primary attention.

Descriptors: Health profile; Inpatient care units; Child.

Con el objetivo de analizar el perfil de los pacientes ingresados en la unidad pediátrica de un hospital universitario, se hizo un estudio descriptivo, retrospectivo que incluyó a los niños hospitalizados en Enfermería Pediátrica y el Pronto Socorro Infantil. Los datos se obtuvieron de los registros de las evaluaciones llevadas a cabo entre mayo y octubre de 2012. Se evaluaron 404 niños, de éstos 35\% son los lactantes y 59\% hombres. Mayo fue el mes con mayor incidencia de hospitalizaciones (21,5\%) y el 58\% eran niños procedentes de Uberaba, Minas Gerais, Brasil. Cuanto al número de residentes habían una frecuencia de cuatro personas / hogar (31,6\%), y la prevalencia de la renta de dos salarios mínimos $(33,6 \%)$ y del número total de niños, el $38 \%$ recibe algún tipo de beneficio. Las enfermedades con mayor incidencia están relacionados con el tracto respiratorio $(28,2 \%)$. Los datos permite caracterizar la muestra, la comprensión del perfil de la enfermedad en este grupo de edad, así como desarrollar planes de cuidados de salud para prevenir el empeoramiento de la enfermedad para que se evite hospitalizaciones futuras, lo que sugiere un enfoque en la prevención de las afecciones respiratórias en la atención primaria. Descriptores: Perfil de salud; Unidades de internación; Niño.

\footnotetext{
${ }^{1}$ Fisioterapeuta. Especialista em Saúde da Criança e Adolescente na modalidade Residência Integrada Multiprofissional em Saúde. Mestranda do Programa de Pós-Graduação em Saúde, Interdisciplinaridade e Reabilitação da Universidade Estadual de Campinas. maraujo.danielle@gmail.com

2 Terapeuta Ocupacional. Especialista em Saúde da Criança e Adolescente na modalidade Residência Integrada Multiprofissional em Saúde. nasegava@gmail.com

3 Educadora Física. Especialista em Saúde da Criança e Adolescente na modalidade Residência Integrada Multiprofissional em Saúde. nandinhagpaula@hotmail.com

4 Psicóloga. Especialista em Saúde da Criança e Adolescente na modalidade Residência Integrada Multiprofissional em Saúde. lorenacvidal@gmail.com

5 Assistente Social. Especialista em Saúde Coletiva e Saúde da Família. Especialista em Saúde da Criança e Adolescente na modalidade Residência Integrada Multiprofissional em Saúde. ju.cancado@hotmail.com

6 Nutricionista. Especialista em Saúde da Criança e Adolescente na modalidade Residência Integrada Multiprofissional em Saúde. jordana_25ma@hotmail.com

${ }^{7}$ Fisioterapeuta. Mestre e Doutora em Ciências da Saúde. Pós Doutorado em Ciências da Saúde. anapaulaespindula@yahoo.com.br
} 


\section{INTRODUÇÃO}

A infância é um período de grande importância no processo de crescimento e desenvolvimento do ser humano, tanto nos aspectos biológicos quanto psicossociais e cognitivos. E o processo de adoecimento em si é um evento que causa um impacto profundo no desenvolvimento humano, trazendo múltiplos significados e alterações no curso da vida do sujeito acometido e de sua família ${ }^{1}$.

A criança hospitalizada fica afastada de sua rotina, ou seja, do seu lar, da escola, da convivência com os amigos e seus familiares. A intervenção terapêutica, a doença e o ambiente hospitalar, isto é, a própria estrutura física, as rotinas, o número de pessoal presente no setor ocasionam grandes transformações na vida da criança e de seus familiares, devido a vários motivos. Em meio a isto, a nova condição de saúde gera incertezas, medo da morte, dor, constrangimento, solidão, saudade, além da perda de autonomia sobre o próprio corpo, que é frequentemente submetido a situações invasivas ${ }^{2}$.

Nas prioridades das políticas públicas voltadas à saúde da população destaca-se a atenção integral à saúde da criança por se tratar de um grupo com maior vulnerabilidade a agravos e enfermidades. Com isso, faz-se necessário conhecer, avaliar e melhorar os indicadores, assim como destacar a importância dos serviços de saúde em vigor ${ }^{3}$.

São indicadores de saúde que refletem o nível de vida da população, informações da estrutura da população (sexo, idade, estado civil, religião, estrutura socioeconômica da população, tipo e qualidade das habitações e ambientes domiciliários), coeficientes de mortalidade e suas causas determinantes, padrão de morbidade da população ou da demanda atendida pelos serviços e grau de risco de um evento ou agravo em saúde ${ }^{4}$.

Outro fator refere-se às altas taxas de internações por vezes inerentes aos cuidados da atenção primária, o que pode significar problemas de acesso aos serviços de saúde ou no seu desempenho; desta forma, o monitoramento dessas hospitalizações pode apoiar a tomada de decisão para enfrentar o excesso de internações evitáveis ${ }^{5}$.

Neste sentido, se torna necessário estudar as condições de saúde das crianças a fim de compreender o perfil de adoecimento nessa faixa etária e a organizar a atenção à saúde para que medidas de prevenção aos agravos possam ser realizadas e que hospitalizações sejam evitadas ${ }^{6}$.

Com o intuito de amenizar o quadro de sofrimento apresentado pelas crianças, despertando novas possibilidades, promovendo a superação das dificuldades, a humanização da assistência, a garantia dos direitos e a criação de espaços lúdicos, faz-se necessário que as equipes de saúde ampliem suas percepções e conhecimentos acerca da população atendida. Desta forma, este estudo tem por objetivo traçar o perfil das internações da enfermaria de pediatria e do pronto-socorro infantil de um hospital universitário.

\section{MÉTODO}

Trata-se de um estudo descritivo, temporal e exploratório das internações da Unidade de Pediatria do Hospital de Clínicas da Universidade Federal do Triângulo Mineiro (UFTM). Esse hospital é referência de assistência a crianças de 27 municípios da macrorregião de Uberaba.

Os dados utilizados neste estudo foram retirados dos arquivos de registro da equipe de Residência Multiprofissional Integrada em Saúde (RIMS - modalidade saúde da criança) anexados aos prontuários dos pacientes. Foram analisados os dados coletados entre $1^{\circ}$ maio a 31 de outubro do ano de 2012. Foram estudadas as variáveis referentes à: mês de internação, gênero, faixa etária, procedência, renda, recebimento de benefício, número de moradores por domicílio e diagnóstico clínico.

As informações contidas nos registros de avaliação da equipe da RIMS foram tabuladas a partir de unidades de registro pré-estabelecidas, anotando-se a ocorrência e a frequência em cada dado avaliado, como o mês de internação, além da verificação do mês de maior e menor incidência. Os gêneros 
foram divididos em masculino e feminino. A faixa etária foi subdividida em recém-nascido (0 a 28 dias), lactente (28 dias a 2 anos incompletos), pré-escolar (2 a 7 anos incompletos), escolar (7 a 10 anos incompletos) e adolescentes ou pré-púbere $(>10 \text { anos })^{7}$.

Em relação à procedência foram divididos em procedentes de Uberaba e procedentes de outras cidades. A renda familiar foi verificada por meio da remuneração em número de salários mínimos. Foi contabilizado o número de moradores por domicílio, bem como os internos (moradores) de outras instituições, por exemplo, casas de proteção. Quanto à benefício público foi observado se há recebimento ou não. As doenças foram agrupadas em sistemas, sendo eles: doenças respiratórias, gastrointestinais, ligados a prematuridade, hematológicas, ortopédicas, cardíacas, dermatológicas e outros.

Posteriormente os dados foram analisados por meio da planilha eletrônica,
Microsoft Excel@, sendo apresentado em tabelas e gráficos, contendo a frequência e percentual de todas variáveis questionadas no estudo.

0 presente estudo foi submetido e aprovado pelo Comitê de Ética em Pesquisas da Universidade Federal do Triângulo Mineiro sob o número de protocolo 2609/2013.

\section{RESULTADOS}

Foram analisados registros da equipe da Residência Multiprofissional Integrada a Saúde, do período de $1^{\text {o }}$ de maio a 31 de outubro, realizaram-se 404 avaliações na unidade de pediatria do Hospital de Clínicas da Universidade Federal do Triângulo Mineiro - HC/UFTM. Destes, 59\% foram do gênero masculino e $41 \%$ do gênero feminino. Conforme a Figura 1 observa-se a classificação dos sujeitos conforme a faixa etária e a maior prevalência foram de lactentes, representado por 143 sujeitos.

Figura 1. Faixa etária de usuários internados na unidade de pediatria do HC/UFTM, Uberaba. 2012.

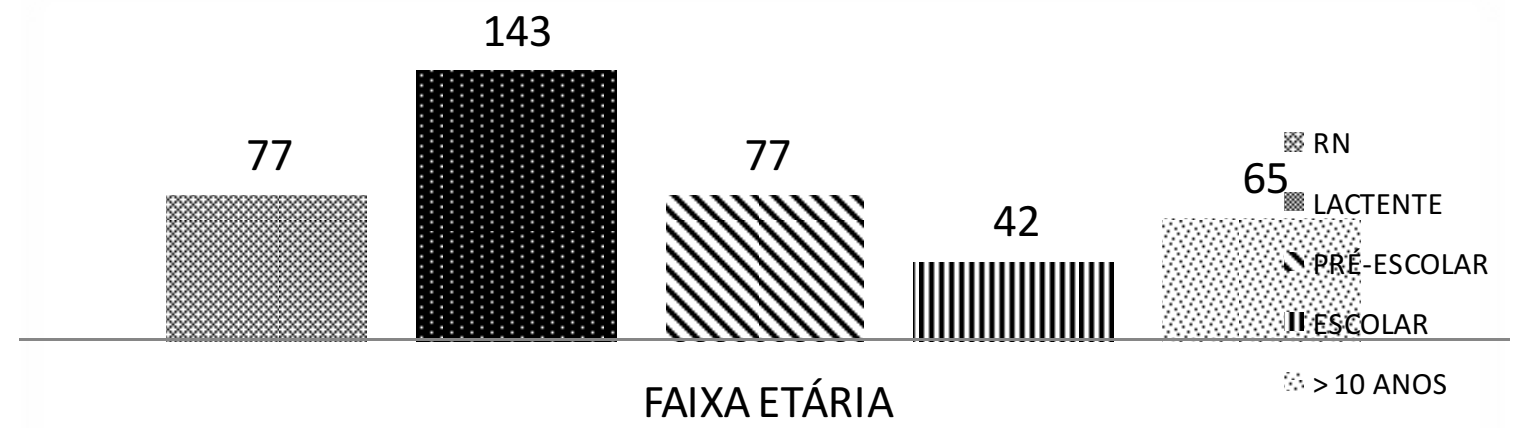

A Tabela 1 apresenta a variedade de casos no total da amostra e foi agrupado segundo diagnóstico da Classificação Internacional de Doenças (CID). As doenças mais prevalentes são as relacionadas ao aparelho respiratório $(\mathrm{n}=114)$, seguida de doenças do aparelho osteoarticular $(n=99)$, prematuridade $\quad(n=64), \quad$ doenças hematológicas $(\mathrm{n}=46)$, doenças do aparelho digestivo $(\mathrm{n}=29)$, doenças do aparelho circulatório $(\mathrm{n}=10)$ e as menos frequentes são as relacionadas ao aparelho tegumentar $(n=5)$. A amostra ainda apresenta sujeitos $(n=37)$ os quais o diagnóstico no momento da triagem multiprofissional ainda não havia sido conclusivo. 
Tabela 1. Crianças atendidas na unidade de pediatria do HC/UFTM segundo diagnósticos agrupados pelo CID, Uberaba, 2012.

\begin{tabular}{lrc}
\hline GRUPOS DO CID & $\mathbf{N}^{\circ}$ & $\mathbf{\%}$ \\
\hline Doenças do aparelho respiratório & 114 & 28,2 \\
\hline Doenças do aparelho osteoarticular & 99 & 24,5 \\
Prematuridade & 64 & 15,8 \\
\hline $\begin{array}{l}\text { Doenças do sangue, Órgãos hematopoiéticos e } \\
\text { Transtornos imunitários }\end{array}$ & 46 & 11,3 \\
Sem informação & 37 & 9,1 \\
\hline Doenças do aparelho digestivo & 29 & 7,1 \\
\hline Doenças do aparelho circulatório & 10 & 2,4 \\
\hline Doenças do aparelho tegumentar & 05 & 1,2 \\
\hline TOTAL & $\mathbf{4 0 4}$ & $\mathbf{1 0 0 , 0}$ \\
\hline
\end{tabular}

Quando analisados os meses de internações, dentre o período delimitado na metodologia (maio a outubro) foi verificado que a maior incidência de internações foi no mês de maio $(21,5 \%)$ e a menor $(9,9 \%)$ no mês de julho.

A maioria das internações (58\%), que são atendidas no HC/UFTM, é proveniente do município de Uberaba (Figura 2). Por sua vez, $42 \%$ das crianças internadas na instituição são oriundos de outras cidades, pois Uberaba é a cidade polo da macrorregião de saúde do Triângulo-Sul e referência para tratamento de alta complexidade de 27 municípios. A formação dessa macrorregião é composta pelas seguintes cidades: Água Comprida, Araxá, Campo Florido, Campos Altos,
Carneirinho, Comendador Gomes, Conceição das Alagoas, Conquista, Delta, Fronteira, Frutal, Ibiá, Itapagipe, Iturama, Limeira do Oeste, Pedrinópolis, Perdizes, Pirajuba, Planura, Pratinha, Sacramento, Santa Juliana, São Francisco de Sales, Tapira, Uberaba, União de Minas e Veríssimo.

Contudo, foi verificado que $11,7 \%$ das cidades atendidas são fora dessa macroregião como: Aguaí $(\mathrm{n}=1)$, Bambuí $(\mathrm{n}=1)$, Igarapava $(n=2)$, Itapira $(n=1)$, João Pinheiro $(n=1)$, Lagoa Verde $(n=1)$, Monte Carmelo $(n=1)$, Nova Ponte $(n=3)$, Nova Serrana $(n=1)$, Presidente Olegário $(n=2)$, Santa Fé $(n=1)$, São Gotardo ( $\mathrm{n}=2)$, São Sebastião do Pontal $(n=1)$, Tiros $(n=1)$, Vazante $(n=1)$.

Figura 2 - Crianças internadas no HC/UFTM segundo procedência. Uberaba, 2012.

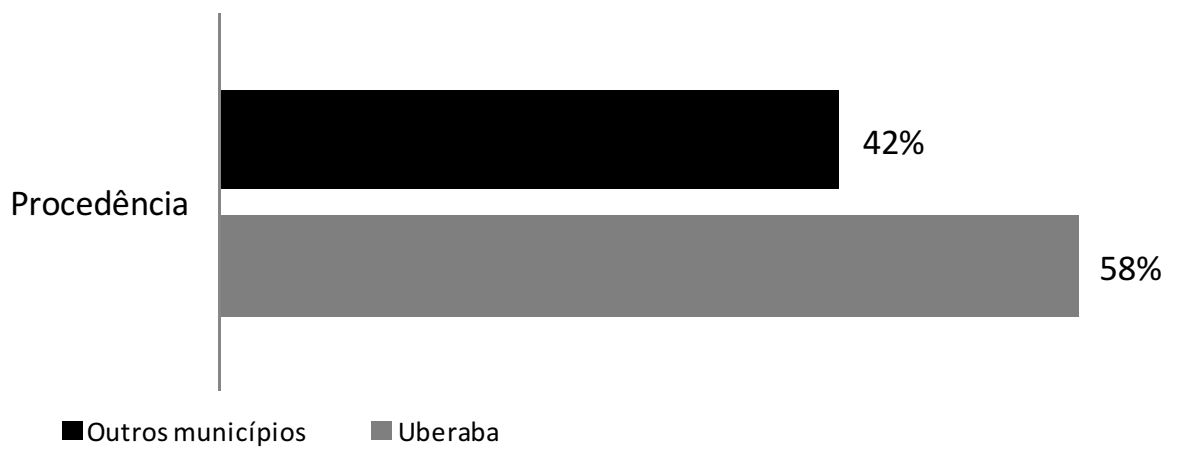

Como demonstrado na Figura 3, os domicílios são compostos na maior parte dos casos por 4 moradores ( $\mathrm{n}=128)$, seguido por $3(n=90)$ e 5 moradores $(n=76)$. Apenas um caso, a criança estava sob guarda de uma casa de proteção (CP). Alguns casos (n=17) a casa era composta apenas por dois moradores, ou seja, a criança e o cuidador. Em dois casos o número de moradores não o número de moradores não foi computado (NC). 
Figura 3. Moradores por domicílio das crianças internadas no HC/UFTM. Uberaba, 2012.

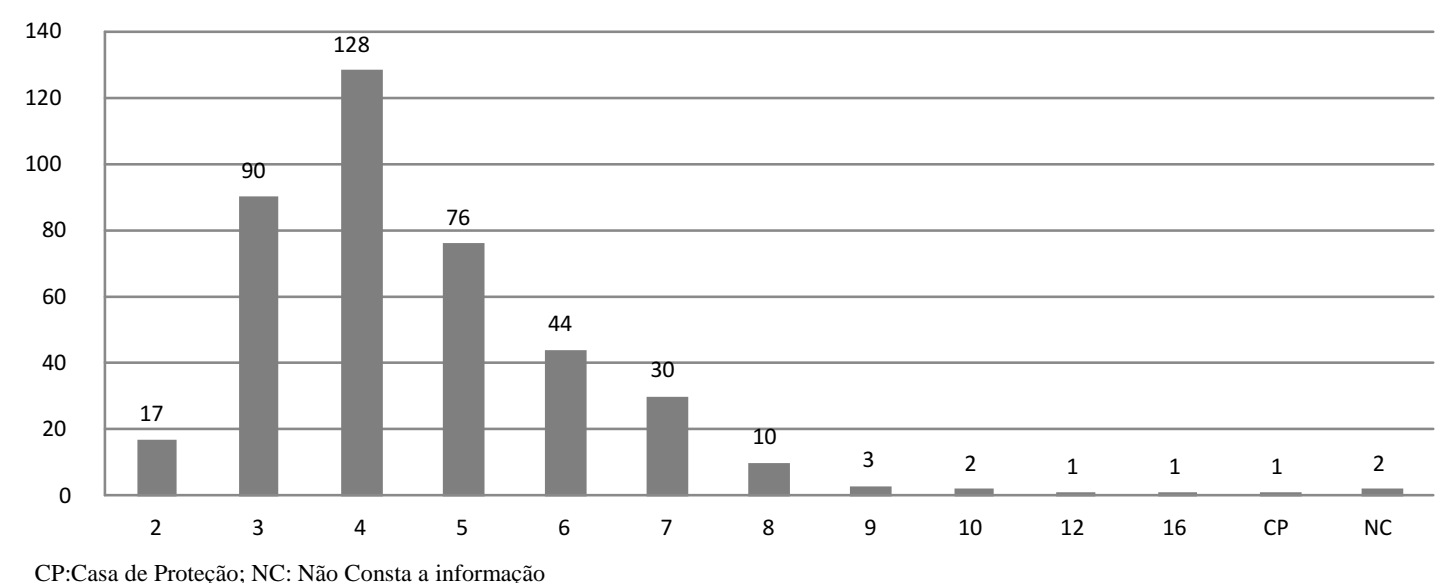

A renda familiar baseada no salário mínimo vigente à época $(\mathrm{R} \$ 622,00)$ e, é apresentada na Figura 4. É possível verificar que a maior parte das famílias possui renda

de 2 salários mínimos $(\mathrm{n}=33,6)$. Das triagens realizadas 38\% das famílias recebem algum tipo de benefício do governo, Bolsa Família ou Benefício de Prestação Continuada.

Figura 4. Renda das famílias de crianças internadas no HC/UFTM. Uberaba, 2012.

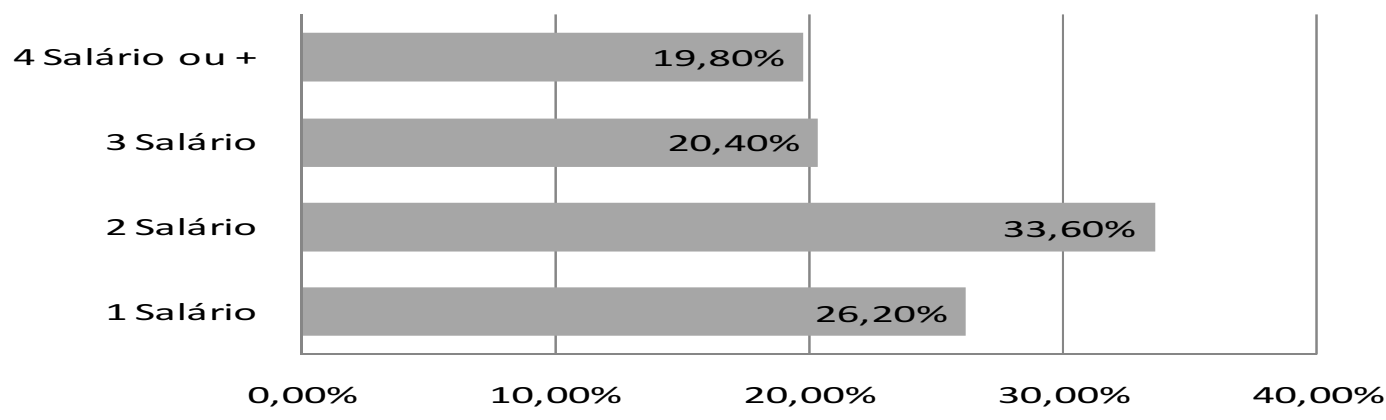

\section{DISCUSSÃO}

Nesse trabalho procurou-se conhecer as características de crianças internadas e avaliadas pela equipe de residentes multiprofissionais na enfermaria pediátrica do Hospital de Clínicas da Universidade Federal do Triângulo Mineiro. Um dado epidemiológico notado no presente estudo e apresentado em outros estudos foi o gênero masculino representar a maior parcela de internações hospitalares. Em concordância com o estudo aqui apresentado estão outras investigações ${ }^{8-14}$ na qual as prevalências de internação pelo sexo masculino foram maiores.

Outro dado epidemiológico relevante é a idade e neste levantamento a mais frequente na enfermaria pediátrica foi a de lactentes. Outros estudos apontam que essa faixa etária está presente entre os maiores índices de internações $8,10,12,13,15,16$. Portanto pode-se inferir a predisposição para 0 adoecimento nessa idade e, com isso alertar os profissionais de saúde quanto ao cuidado desse público.

Quanto às causas responsáveis pela internação, destacam-se as afecções respiratórias, que representaram 28,2\%. Outros hospitais universitários apresentam taxas ainda maiores. No Hospital Universitário Clemente de Faria a frequência de internações por doenças respiratórias foi de $36,5 \%{ }^{10}$, no Hospital da Universidade Federal do Mato Grosso do Sul foi $42,8 \% 13$ e no Hospital da Universidade Federal de Santa Catarina foi de $44,8 \%^{8}$. No Hospital de São Luis (MA), a pneumonia destacou-se entre as afecções respiratórias, sendo ela de 15,56\%14. Em contrapartida, no Hospital Escola da Universidade Federal de Pelotas a 
principal causa foi relacionada às doenças do recém-nascido, representado por $27,4 \%$ e as doenças respiratórias ficaram em segundo lugar com 25,5\%12. Em um estudo semelhante realizado na Índia, as causas de internação se balancearam entre afecções gastrointestinais e respiratórias, cada uma delas com $23 \%{ }^{16}$.

Em relação às cidades de procedência, verifica-se que $42 \%$ não são de Uberaba, e destes $11,7 \%$ não fazem parte da macrorregião. 0 grande índice de cidades atendidas fora da área de abrangência da macrorregional Uberaba pode ser justificado pelo funcionamento do Sistema Estadual de Regulação Assistencial (SUS Fácil), que por meio da instrumentalização dos fluxos garante a população o acesso aos serviços de saúde do SUS ${ }^{17}$. Outra forma de acesso pode ter ocorrido por meio da CNRAC (Central Nacional de Regulação de Alta Complexidade), responsável pela referência interestadual de pacientes, que necessitam de atendimento de alta complexidade, de caráter eletivo, para outro Estado ${ }^{18}$.

A baixa renda e a alta densidade familiar encontrada no estudo podem estar relacionadas às internações principalmente quando se observa a faixa etária mais prevalente. Mais precisamente em relação às afecções respiratórias a alta densidade familiar pode ser uma das causas, além de presença de fumantes no domicílio e daquelas crianças cuidadas pelas avós ${ }^{19,20}$.

Quanto ao período de internação mais frequente foi encontrado que o mês de maio (outono) obteve mais registros de internação. Tal fato não entra em concordância com outros estudos na qual o período de maior internação é no inverno ${ }^{21,22}$. Não foi possível relacionar com o nosso estudo, pois se tratou de uma análise epidemiológica semestral.

\section{CONCLUSÃO}

Por meio do conhecimento epidemiológico das crianças avaliadas na enfermaria de pediatria e emergência do HC/UFTM é possível realizar uma adequação dos serviços prestados, podendo melhorar o atendimento de modo que a população seja beneficiada. Além disso, espera-se que seja um embasamento para a realização de ações de promoção da saúde e prevenção de doenças, fazendo com que os usuários recebam um atendimento integral.

Com isso, faz-se necessário intensificar o atendimento à criança na atenção primária prevenindo as internações, e também, proporcionar esclarecimentos aos pais e responsáveis pelas crianças sobre o cuidado com a saúde delas. E sempre realizar e incentivar a educação permanente para os profissionais de saúde que atuam nessa unidade, proporcionando condições para realizar assistência com qualidade.

\section{REFERÊNCIAS}

1. Silva DF, Corrêa I. Reflexão sobre as vantagens, desvantagens e dificuldades do brincar no ambiente hospitalar. REME Rev Min Enferm. 2010; 14(1):37-42.

2. Parcianello AT, Felin RB. E agora doutor, onde vou brincar? Considerações sobre a hospitalização infantil. Barbarói. 2008; 28:147-66.

3. Retrão MMS, Oliveira EAR, Lima LHO, Duailibe FT, Silva RN, Brito BB. Hospitalizações de menores de cinco anos em hospital público: um estudo descritivo. Rev Interdiscip. 2013; 6(3):143-51.

4. Teodoro BN, Andrade SM. Internações pediátricas no hospital universitário regional norte do Paraná, 1998. Espaç Saúde. 2000; 1:89-112.

5. Barreto JOM, Nery IS, Costa MSC. Estratégia Saúde da Família e internações hospitalares em menores de 5 anos no Piauí, Brasil. Cad Saúde Pública. 2012; 28(3):515-26.

6. Oliveira BRG, Viera CS, Collet N, Lima RAG. Causas de hospitalização no SUS de crianças de zero a quatro anos no Brasil. Rev Bras Epidemiol. 2010; 13(2):268-77.

7. Molina RCM, Marcon SS, Uchimura TT, Lopes EP. Caracterização das internações em uma unidade de terapia intensiva pediátrica, de um hospital-escola da região sul do Brasil. Cienc Cuid Saude. 2008; 7(1):112-20.

8. Silvério A. Perfil da clientela do serviço de emergência pediátrica do Hospital Universitário da UFSC. ACM Arq Catarin Med. 1996; 25(4):311-5. 
9. Caldeira T, Santos G, Pontes E, Dourado R, Rodrigues L. O dia-a-dia de uma urgência pediátrica. Acta Pediatr Port. 2006; 1(37):14.

10. Sena RR, Leite CR, Santana JJF, Vieira MA. Perfil das crianças atendidas na unidade de pediatria do Hospital Universitário Clemente de Faria, Montes Claros - MG. Unimontes Cient. 2006; 8(1):117-28.

11. Veras JEGLF, Carvalho AT, Uchôa JL, Nascimento LA, Almeida PC, Ximenes LB. Profile of children attended by according to emergency rating: documental study. Online Braz J Nurs. (Online). 2011 [citado em 12 ago 2013]; 10(3). Disponível em: http://www.objnursing.uff.br/index.php/nur sing/article/view/3264/1149.

12. Granzotto JA, Fonseca SS, Steffen MS, Machado MM, Roncaglio R, Lima DP, et al. Fatores relacionados à internação pediatrica em um hospital universitário da região sul do Brasil. Pediatria (São Paulo). 2010; 32(1):159.

13. Salgado RMP, Aguero FCM. Perfil dos pacientes pediátricos atendidos na emergência de um hospital universitário. Pediatria (São Paulo). 2010; 32(2):90-7.

14. Ribeiro TSF, Fonseca MSS, Sousa NVS, Queiroz RCCS, Bezerra MLL, Queiroz LLC. Prevalência de internações em crianças de 02 anos em um hospital de referência, São Luis - MA, 2012. Rev Ciênc Saúde. 2012; 14(2):127-32.

15. Goh AY, Chan TL, Abdel-Latiff ME. Paediatric utilization of a general emergency department in a developing country. Acta Paediatr. 2003; 92(8):965-9.

16. Singhu S, Jain V, Gupta G. Pediatric emergencies at a tertiary care hospital in India. J Trop Pediatr. 2003; 49(4):207-11.
17. Sistema Estadual de Regulação Assistencial de Minas Gerais. SUS Fácil MG: central de regulação. Belo Horizonte: Secretaria de Estado da Saúde de Minas Gerais; 2005 [citado em 31 ago 2013]. 40p. Disponível em: http://www.mp.go.gov.br/portalweb/hp/2/ docs/cartilha_sistema_estadual_regulacao_as sistencial_mg.pdf.

18. Junior VCP, Oliveira Fraga MN, Freitas SM. Análise das portarias que regulamentam a Política Nacional de Atenção Cardiovascular de Alta Complexidade. Rev Bras Cir Cardiovasc. 2012; 27(3):463-8.

19. Jackson S, Mathews $\mathrm{KH}$, Pulanić $\mathrm{D}$, Falconer R, Rudan I, Campbell H, Nair H. Risk factors for severe acute lower respiratory infections in children-a systematic review and meta-analysis. Croat Med J. 2013; 54(2):110-21.

20. Matijasevich A, César JÁ, Santos IS, Barros AJD, Dode MASO, Barros FC, et al. Internações hospitalares durante a infância em três estudos de base populacional no Sul do Brasil: tendências e diferenciais. Cad Saúde Pública. 2008; 24(3):437-43.

21. Holman RC, Shay DK, Curns AT, Lingappa JR, Anderson LJ. Risk factors for bronchiolitisassociated deaths among infants in the United States. Pediatr Infect Dis J. 2003; 22:483-90.

22. Veras TN, Sakae TM. Características de crianças hospitalizadas com asma grave no sul do Brasil. Sci Med. 2010; 20(3):223-7.

CONTRIBUIÇÕES
Todas as autoras participaram do
delineamento do estudo, coleta e tabulação
dos dados, análise, interpretação e redação
do manuscrito. Ana Paula Espíndula
coordenou a pesquisa e fez a revisão do texto
final do manuscrito.

